\title{
COVID-19 Infection Rate among Dental Health Care Workers
}

\author{
Findler $\mathbf{M}^{1}$, Moritz $\mathrm{K}^{1}$, Rosen $\mathrm{R}^{1}$, Bernstein $\mathrm{Y}^{1}$, Mann $\mathrm{J}^{1}$ and Tobias $\mathrm{G}^{2 *}$ \\ ${ }^{1}$ Dental Research Unit-MaccabiDent, Maccabi Healthcare Fund, Israel \\ ${ }^{2}$ Department of Community Dentistry, Hebrew University-Hadassah School of Dental Medicine, \\ Israel
}

*Corresponding author: Guy Tobias, Department of Community Dentistry, Hebrew UniversityHadassah School of Dental Medicine, Hadassah Medical Center Ein Kerem, Jerusalem, Israel, Tel: 972527052333; Email: guy.tobias@mail.huji.ac.il

\section{Research Article}

Volume 6 Issue 1

Received Date: January 11, 2021

Published Date: January 25, 2021

DOI: $10.23880 /$ oajds-16000285

\section{Abstract}

Background: First infections of COVID-19 pandemic occurred in December 2019 in China and then spread rapidly all over the world. In February 2020, the first Corona case was diagnosed in Israel, since then, three national lockdowns have been imposed. Israeli Ministry of health published guidelines for dental treatment during times of social distancing which include: 1) Questioning patients prior to admittance. 2) Increasing intervals between appointments. 3) Restricting number of people permitted in the waiting room to prevent crowds. 4) Wearing masks at all times. 5) Wearing long sleeve waterproof surgical gowns, goggles / face shield.

Methods: Data from 54 dental clinics (MaccabiDent health maintenance organization) were collected between March and October 2020 regarding: 1) Number of treatments performed. 2) Number of health care providers participating in the procedures. 3) Number of patients with positive COVID-19 results. 4) Number of healthcare workers positive for COVID-19. 5) Number of paramedical and administrative staff positive for COVID-19.6) Number of patients treated by dentists who later turned out to be infected by the virus.

Results: 1,079 dentists and 354 dental hygienists worked during the study period, and performed 1.29 million procedures of these, seventy eight dentists treated 99 verified COVID-19 patients. 17 dentists (1.58\%) tested positive for the virus.

Conclusion: By following guidelines, the risk of transmission of coronavirus is minimal in the dental setting.

Practical Implications: This article reinforces the evidence regarding the importance of education and following guidelines regarding infection control for dental professionals employed in public clinics.

Keywords: COVID-19; Infection control

Abbreviations: HMO: Health Maintenance Organizations; PPE: Personal Protective Equipment

\section{Introduction}

The COVID-19 virus enters the body through the respiratory system, i.e. the nose and mouth, are the portal of entry. The oral cavity has been found to be the primary reservoir of the virus [1]. Dental treatment using aerosolforming equipment exposes the dentist and clinical staff to the virus, particularly since the dentist sits at a distance of $20-40 \mathrm{~cm}$ from the patient's uncovered mouth [2]. Maccabi health care fund is one of four health maintenance organizations (HMO) operating in Israel, and has about 2.5 million members eligible for dental care in MaccabiDent, the HMO's network of dental clinics [3]. MaccabiDent has 
54 dental clinics with 346 dental units operated by 1,079 dentists and 354 dental hygienists.

On February 21, 2020, the first patients with COVID-19 were identified in Israel. On March 15, the first national lockdown was imposed and the infection rate peaked on March 31 and then rapidly declined. The numbers began to rise in late May and peaked on September $23^{\text {rd }}$, the 2nd lockdown began on September $18^{\text {th }}$, after which the infection indices fell [4] the third lockdown was imposed on December 27th. During this period, the Israel Ministry of Health published clinical guidelines for dental treatment. According to the initial guidelines, only emergency dental treatments should be performed and patients that would suffer from future dental deterioration should also receive treatment. Since the onset of the epidemic, distancing procedures and use of Personal protective equipment (PPE) in dental clinics have been implemented to prevent the spread of infection.

\section{Methods}

We collected all the data regarding dental treatments, COVID-19 infections of health care workers, and possible COVID-19 infections in patients for the 7 month period between March 19 and October 19, 2020. Data was collected weekly directly from MaccabiDent's 54 dental clinics, and summarized by the epidemiological nurse at MaccabiDent headquarters. The data was transferred to the Israel Ministry of Health, the management of Maccabi Dent, and the clinical research unit. The data included:

a) Number of treatments performed.

b) Number of health care providers participating in the procedures.

c) Number of patients with positive COVID-19 results.

d) Number of healthcare workers positive for COVID-19.

e) Number of paramedical and administrative staff positive for COVID-19.

f) Number of patients treated by dentists who later turned out to be infected by the virus.

\section{Results \& Discussion}

1,079 active dentists, 354 dental hygienists, 818 dental assistants, and 508 administrative staff are employed at MaccabiDent. There are 389 dental units in 54 clinics nationwide. A total of 1.29 million dental procedures were performed during this period. Seventy eight dentists treated 99 verified COVID-19 patients, and 17 dentists (1.58\%) were found positive for COVID-19. Retrospective epidemiological studies showed that only 2 dentists $(0.185 \%)$ treated patients who were positive for COVID-19. Since both treatments were performed within a week of the dentist's infection, it is likely that they contracted the virus during the treatment. Three dental hygienists $(0.85 \%)$, nine dental assistants $(1.1 \%)$, and three administrative staff $(0.59 \%)$ were positive for the virus so that 32 MaccabiDent workers $(1.2 \%)$ were positive for COVID-19 (flow chart).

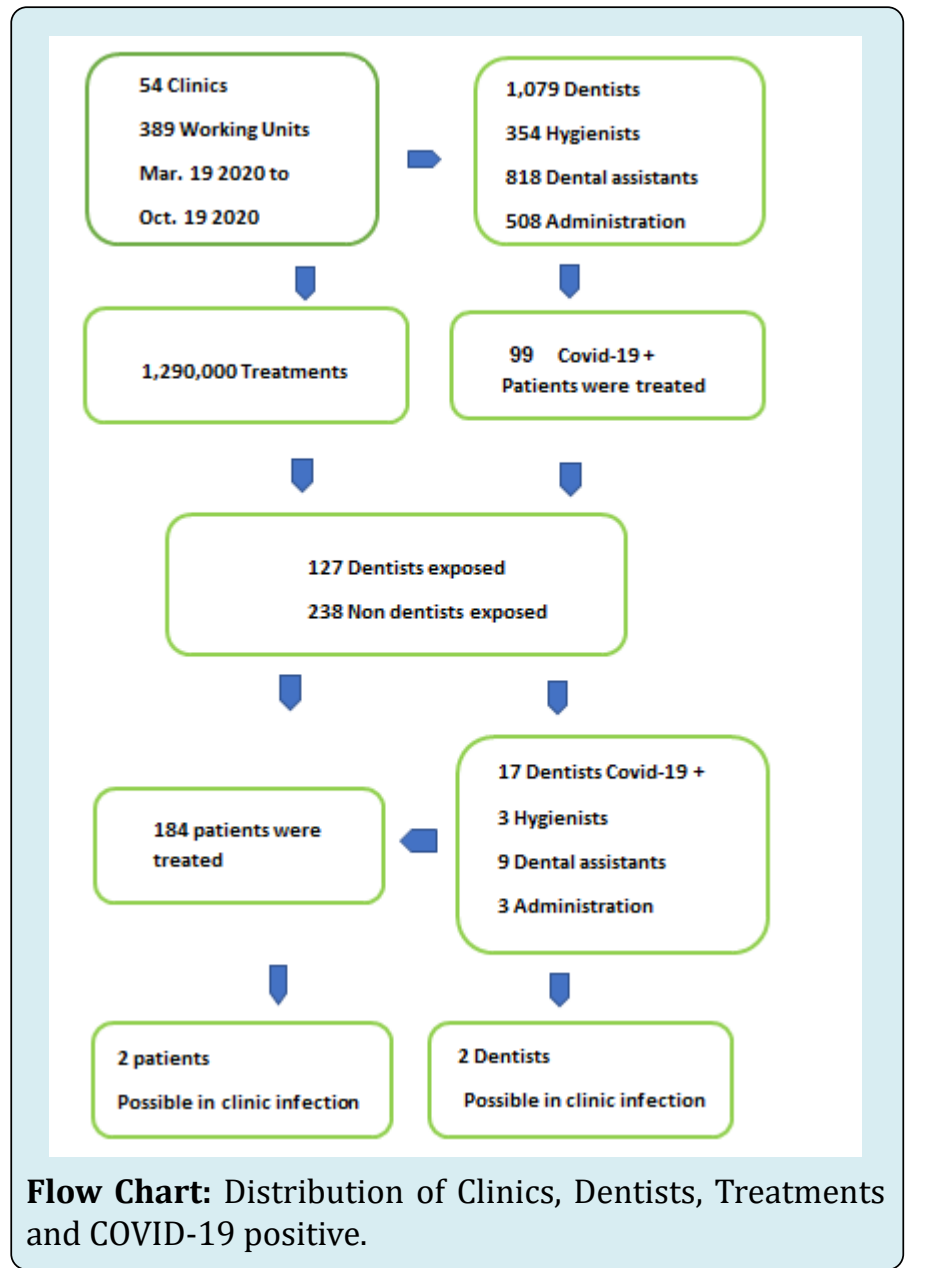

184 dental procedures were performed on 99 patients by dentists who were retrospectively diagnosed as positive for the coronavirus. Only two patients were retrospectively found to be positive for the coronavirus. It is not clear whether they became infected as a result of the treatment or were infected elsewhere.

\section{Conclusion}

COVID-19 is a contagious disease that has caused a pandemic. Due to the highly infectious nature of this virus, health care professionals are committed to continuing treating their patients while minimizing infection transmission. When it comes to COVID-19, the main sites of entry of Sars-cov2 to the body are the nose and mouth. Thus, dental treatments are high-risk procedures due to the combination of working on an uncovered mouth with spray forming equipment, for a significant period of time. 
The level of infection among dentists was found to be $0.185 \%$, and the level of COVID-19 verified company employees was $0.53 \%$, resembling the infection rates in health care workers in the Philippines $0.085 \%$ (low level of disease). Much higher infection rates of $2.61 \%$ and $3.44 \%$ were found among medical workers in Italy and in Spain [5] with a relative high rate of death [6]. Dental care is often required to alleviate pain, remove foci of infection, and restore the chewing mechanism. Routine dental care often involves many different types of treatment and laboratory work. Discontinuing therapy for long periods causes irreversible damage to the patient. Thus, dentists are required to continue to provide routine care and emergency treatments. The role of the medical system and its managers is to take all necessary precautionary measures to minimize infection transmission between staff and patients. To this end, it is important to facilitate the performance of treatments in a safe environment [7]. The level of infection in the dental clinics was $0.185 \%$ (low), probably due to the provision of adequate protection.

In the SARS epidemic, there were high infection rates among health care workers, with a mortality rate of about $10 \%$. The total number of victims of the epidemic globally was 8,096, of whom 774 died, and approximately one third of the casualties were medical workers [8]. Based on our data, we can conclude that with proper personal protective equipment the staff in the dental clinics are at a low risk of infection from the virus, at levels similar to the medical staff in countries where the level of morbidity from the virus is low.

\section{References}

1. Wiersinga WJ, Rhodes A, Cheng AC, Peacock SJ, Prescott HC (2020) Pathophysiology, Transmission, Diagnosis, and Treatment of Coronavirus Disease 2019 (COVID-19): A Review. JAMA 324(8): 782-793.

2. Estrich CG, Mikkelsen M, Morrissey R, Geisinger ML, Ioannidou E, et al. (2020) Estimating COVID-19 prevalence and infection control practices among US dentists. J Am Dent Assoc 151(11): 815-824.

3. Findler $M$, Bernstein $Y$, Meidan Z, Tobias G, Manor L, et al. (2020) Dental emergencies during the COVID-19 aversion therapy centers. Open Access J Dental Sciences 5(4): 1-5.

4. https://newmedia.calcalist.co.il/data_journalism/ corona/index.html

5. Armocida B, Formenti B, Ussai S, Palestra F, Missoni E (2020) The Italian health system and the COVID-19 challenge. The Lancet Public Health 5(5): 253.

6. Ing EB, Xu QA, Salimi A, Torun N (2020) Physician deaths from corona virus (COVID19) disease. Occup Med (Lond) $70(5): 370-374$

7. Gianola FJ (2007) The duty to treat and realities of the $21^{\text {st }}$ century. JAAP 20: 48.

8. WHO'S April 21, 2004 concluding report In the SARS epidemic.

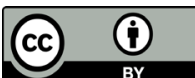

